\title{
Competencia y medidas liberalizadoras en el sector minorista de hidrocarburos: análisis del efecto sobre los precios de la entrada de nuevos operadores en España*
}

\author{
Valeria Bernardo \\ Universitat de Barcelona \\ Juan Luis Jiménez González \\ Universidad de Las Palmas de Gran Canaria \\ Jordi Perdiguero García \\ Universitat Autònoma de Barcelona
}

\section{Resumen}

Años después de la liberalización del sector y de literatura académica evaluatoria postreforma, resulta reiterativo mencionar el reducido nivel de competencia existente en el mercado de carburantes de España. En el presente trabajo mostramos un análisis histórico de los casos de competencia analizados por los diversos organismos encargados de velar por ella en España para, a partir de ahí, describir las políticas llevadas a cabo por el Estado para tratar de mejorar la ausencia de tensión competitiva entre las empresas. Centrándonos en una de las últimas medidas llevadas a cabo (2013), y aplicando un análisis econométrico con datos detallados para la Región Metropolitana de Barcelona, nuestros resultados apuntan a precios de los entrantes menores a la media entre 5 y 15 céntimos, principalmente estaciones «low cost» y de supermercados. No obstante, estos efectos positivos pueden reducirse en áreas de baja densidad poblacional.

Palabras clave: hidrocarburos, competencia, España

Clasificación JEL: L13, L41, L52.

\section{Abstract}

It is repetitive mention the low level of competition in the petrol market in Spain. In this paper we present a historical analysis of competition cases analyzed by antitrust and regulatory agencies in Spain, from there, describing the policies pursued by the State to try to improve the lack of competitive tension between companies. Focusing on one of the last measures taken (2013), and applying a detailed econometric analysis for the Metropolitan Region of Barcelona, our results show how prices decrease between 5 and 15 cents after entrants open new petrol stations, mainly «low cost» and supermarkets. However, these positive effects may be reduced in areas of low population density.

Keywords: petrol, competition policy, Spain.

JEL classification: L13, L41, L52.

* Los autores quieren agradecer la invitación a participar en este monográfico por parte de Gustavo Nombela, así como los comentarios recibidos por un evaluador anónimo. Cualquier error es de nuestra entera responsabilidad. 


\section{Introducción}

El sector de los carburantes es un ejemplo «de libro de texto» de mercado con reducida competencia. Y este resultado es compartido por buena parte de la literatura empírica internacional ${ }^{1} \mathrm{y}$, cómo no, también para el caso español ${ }^{2}$. La pregunta inmediata es: ¿por qué sucede esto?

Hasta donde conocemos hay dos visiones complementarias y simples para responder a este resultado de baja competencia a nivel internacional. Desde la perspectiva de la oferta, se debe tener conciencia de la propia concepción seminal anticompetitiva del sector a nivel mayorista. Y nos referimos al paradigma del «cartel de la OPEP» ${ }^{3}$. Por definición, un cartel está prohibido en cualquier legislación en materia de competencia de cualquier país del mundo, menos este caso... Los motivos de tal exención son obvios y subyacen en el contexto del conjunto del mercado, donde precisamente constituyen un ejemplo para el sector aguas abajo.

Pero es que desde la perspectiva de la demanda, las características del comportamiento apuntan a la misma dirección. Ésta cumple todos los factores necesarios para facilitar las actividades de colusión y, en general, de comportamientos anticompetitivos: inelasticidad-precio de la demanda, baja sustituibilidad con otros productos, reducida innovación de producto, estabilidad en cuotas de mercado, etcétera (véase Ivaldi et al., 2003).

En este marco facilitador de comportamientos anticompetitivos, todo el sector productivo presenta problemas de competencia que, en última instancia, se traducen en precios minoristas más elevados de los que existirían de no ser por ello. En España, y a pesar de las casi tres décadas de liberalización del mercado peninsular -la experiencia canaria es más longeva, aunque los resultados no son ejemplares desde la perspectiva minorista, como apuntan Perdiguero y Jiménez, 2009-, los últimos informes de la extinta Comisión Nacional de la Competencia (2012) y Comisión Nacional de la Energía (2013), así como los expedientes abiertos desde la liberalización, son indicadores evidentes de los problemas que el sector muestra.

El presente trabajo tiene dos objetivos: el primero es describir sucintamente los expedientes en materia de competencia del sector, como aquellas políticas implementadas en las dos últimas décadas para liberalizar efectivamente el mercado. Y el segundo objetivo es precisamente evaluar una de esas políticas, concretamente el Real Decreto 04/2013 que permitía un acceso más laxo de estaciones de servicio en polígonos industriales, entre otros. Nuestros resultados apuntan a reducciones en precios, pero con matices.

\footnotetext{
${ }^{1}$ Por ejemplo, véase ECKERT (2013) para un survey internacional acerca de los problemas de competencia en el mercado minorista.

${ }^{2}$ Para un análisis reciente del caso español, los estudios previos realizados o un indicador de falta de competencia denominado «el efecto lunes», véase BELLO y CAVERO (2008), PERDIGUERO (2012) y JIMÉNEZ y PERDIGUERO (2013 y 2014).

${ }^{3}$ Aunque los países integrantes de la Organización de Países Exportadores de Petróleo (OPEP), no son los mayores productores (extractores) actuales de petróleo, cuentan con una cuota relevante en el comercio internacional y es un patrón de comportamiento que evidencia las rigideces del sector.
} 
A partir de la presente Introducción, el artículo se estructura de la siguiente manera. En la sección 2 se efectúa un recuento de los expedientes de competencia a los que ha estado sometido el sector durante los últimos 15 años y de las regulaciones favorecedoras de la competencia sancionadas desde el año 1985. La sección 3 contiene la evaluación de los efectos del Real Decreto 04/2013, que se estructura en dos subsecciones: por un lado se presenta la base de datos, y, por otro, la metodología utilizada y los resultados. Finalmente, el artículo cuenta con un apartado de conclusiones.

\section{Aplicación de la competencia y normas favorecedoras de la misma}

Como todo sector en España, la venta mayorista, minorista y actividades relacionadas con los carburantes se encuentra sujeto a la aplicación de la normativa en materia de Competencia (actualmente la Ley 15/2007), además de la normativa específica que le es de aplicación. En los últimos 15 años (esto es, prácticamente desde el proceso de liberalización sectorial), los diferentes organismos encargados de velar por la competencia en España (véase el artículo de Borrell, Jiménez y García en este mismo número, para entender el marco institucional), han abierto diversos expedientes y tratado varios procesos de concentración en el sector.

La información relativa a dichos expedientes se encuentra someramente resumida en el Cuadro 1. De ella podemos destacar varias cuestiones. En primer lugar, que hay una media de más de un expediente por año (lo que habría que comparar con otros sectores aunque, a priori, dudamos de mayores valores a éste). En segundo término, el 60 por 100 de los expedientes abiertos tienen relación con una práctica anticompetitiva. Para los procesos de concentraciones, se observa cómo la mayoría se concentran en el periodo de crisis económica.

Y por último, reseñar la prolija utilización que las empresas han hecho del derecho a recurso sobre las sentencias del Tribunal de Defensa de la Competencia y la Comisión Nacional de la Competencia, alargando en algunos casos el proceso más de una década y, en buena parte de ellos, dictaminando resoluciones por la Audiencia Nacional y/o el Tribunal Supremo contrarias a las primeras. Estos resultados, lícitos (obviamente), debilitan uno de los pilares de todo sistema de competencia: la capacidad de disuasión del mismo.

Por otra parte, el Sector Público no sólo realiza actividades de disuasión, detección y sanción, sino que procura el establecimiento de normas que faciliten la libre entrada y, en general, la competencia efectiva en el mercado. Dados los resultados del mercado y a pesar de las medidas llevadas a cabo en los tres últimos lustros, éstas parecen no haber tenido un éxito destacable (véase Cuadro 2 para una cronología y descripción de las mismas).

La mayor parte de la actividad normativa en este sector se dio, principalmente, en la década de los 90. Posteriormente, tras eliminar la regulación de distancias mínimas entre estaciones, determinados aspectos de carteles y la regulación de precios 


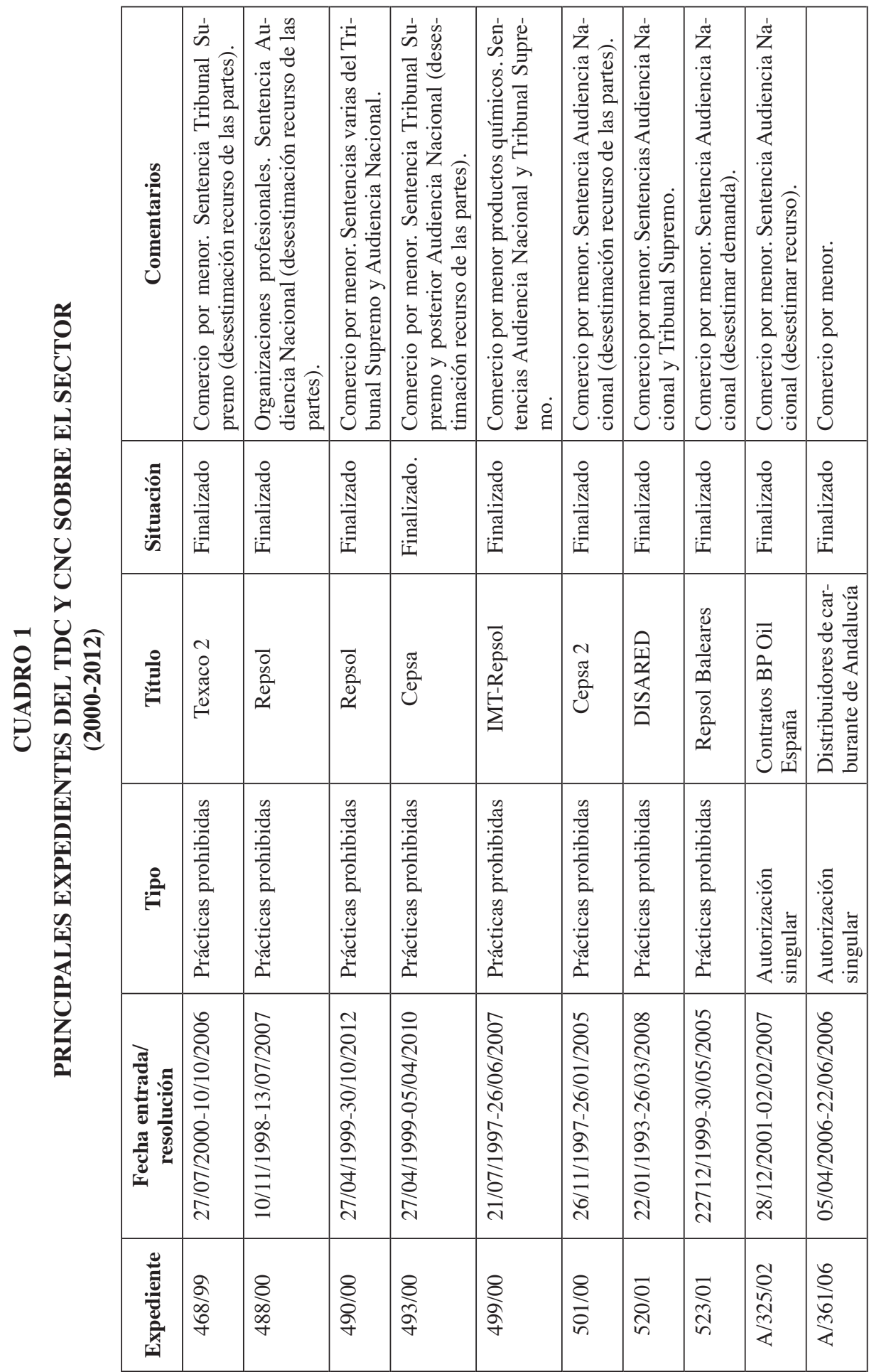




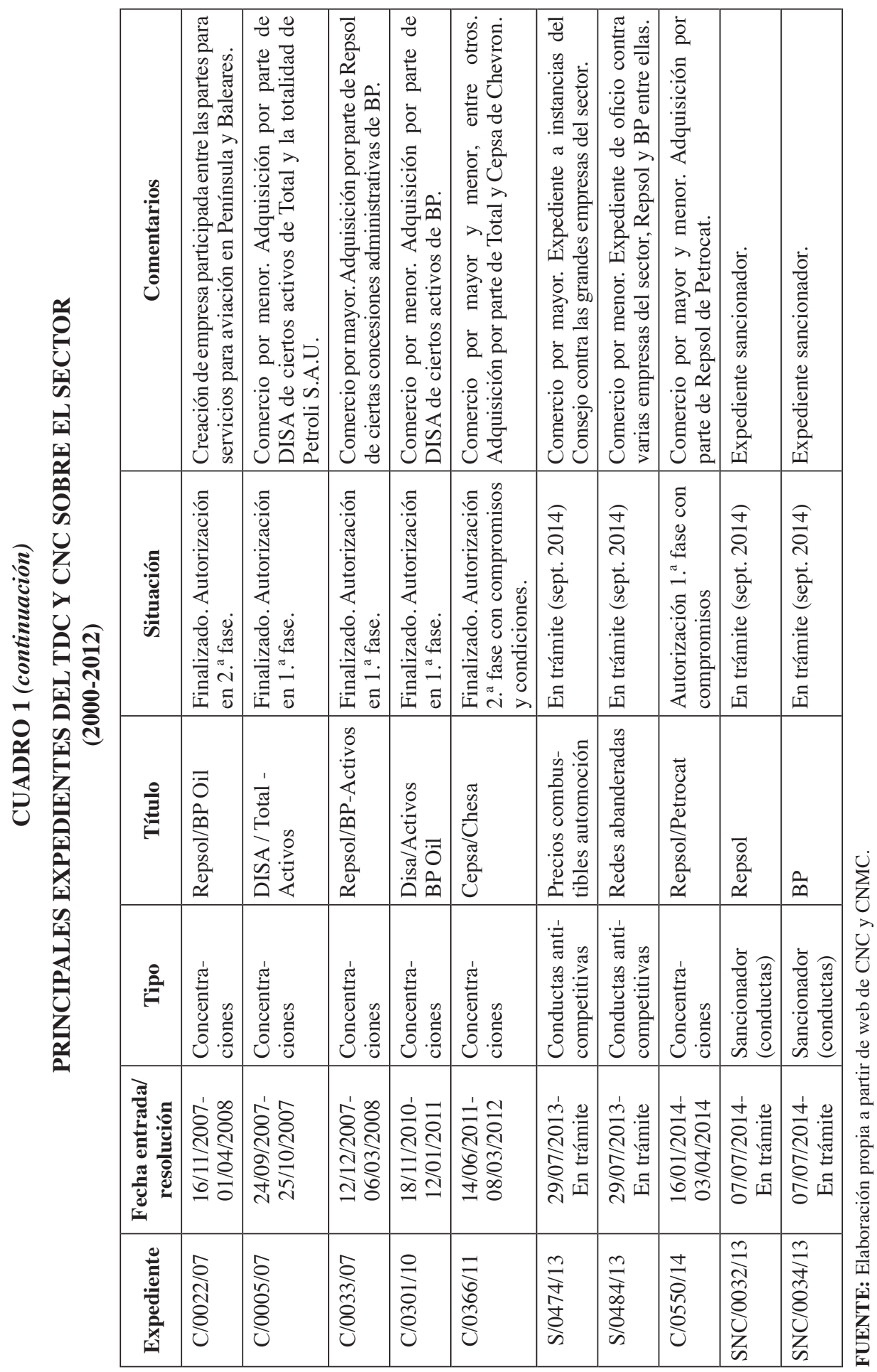




\section{CUADRO 2}

\section{PRINCIPALES NORMATIVAS LIBERALIZADORAS SOBRE EL SECTOR} (1985-2013)

\begin{tabular}{|c|c|c|}
\hline Año & Normativa & Principales medidas favorecedoras de la competencia \\
\hline 1985 & $\begin{array}{l}\text { Real Decreto-Ley } \\
5 / 1985 \text {, de } 12 \text { de } \\
\text { diciembre }\end{array}$ & $\begin{array}{l}\text { Apertura a las importaciones y exportaciones de productos petro- } \\
\text { líferos de otros países de la CEE limitada según convenios. Se } \\
\text { establece como fecha de liberalización total el } 1 \text { de enero de } 1992 . \\
\text { Se permite la creación de una red de distribución minorista para } \\
\text { los productos importados de la CEE paralela a la red concesional } \\
\text { del Estado. } \\
\text { Se liberaliza la importación de crudo. }\end{array}$ \\
\hline 1988 & $\begin{array}{l}\text { Real Decreto-Ley } \\
4 / 1988 \text {, de } 24 \text { de junio }\end{array}$ & Reducción de las distancias mínimas entre estaciones de servicio. \\
\hline 1990 & $\begin{array}{l}\text { Orden de } 6 \text { de julio de } \\
1990\end{array}$ & $\begin{array}{l}\text { Establecimiento de un sistema de precios máximos para las } \\
\text { gasolinas y gasóleos de automoción, que reemplaza al sistema de } \\
\text { precios fijos, en el ámbito de la Península y Baleares. }\end{array}$ \\
\hline \multirow[t]{2}{*}{1991} & $\begin{array}{l}\text { Orden de } 3 \text { de mayo } \\
\text { de } 1991\end{array}$ & $\begin{array}{l}\text { Establecimiento de un sistema de precios máximos para las gaso- } \\
\text { linas y gasóleos de automoción, que reemplaza al sistema de pre- } \\
\text { cios fijos, en el ámbito de las Islas Canarias. }\end{array}$ \\
\hline & $\begin{array}{l}\text { Real Decreto-Ley } \\
\text { 2/1991, de } 29 \text { de } \\
\text { noviembre }\end{array}$ & $\begin{array}{l}\text { Autorización de la escisión de CAMPSA en varias empresas } \\
\text { participadas por firmas refinadoras y eliminación de CAMPSA } \\
\text { como único intermediario para la venta de productos petrolíferos. } \\
\text { Reducción de las distancias mínimas entre estaciones de ser- } \\
\text { vicio. }\end{array}$ \\
\hline 1992 & $\begin{array}{l}\text { Ley } 34 / 1992 \text {, de } 22 \text { de } \\
\text { diciembre }\end{array}$ & $\begin{array}{l}\text { Extinción del monopolio de petróleos. } \\
\text { Liberalización de actividades de importación, exportación y de } \\
\text { intercambio intracomunitario de crudo de petróleo y productos } \\
\text { petrolíferos, su refino, distribución, venta, transporte y almacena- } \\
\text { miento. } \\
\text { Eliminación de la distinción entre red concesional y paralela. } \\
\text { Establecimiento de la libre elección de suministrador por parte de } \\
\text { las estaciones de servicio. }\end{array}$ \\
\hline 1995 & $\begin{array}{l}\text { Real Decreto } 155 / 1995, \\
\text { de } 3 \text { de febrero }\end{array}$ & $\begin{array}{l}\text { Supresión del régimen de distancias mínimas entre estableci- } \\
\text { mientos. }\end{array}$ \\
\hline 1996 & $\begin{array}{l}\text { Orden Ministerial de } \\
10 \text { de junio de } 1996\end{array}$ & $\begin{array}{l}\text { Liberalización de los precios de los gasóleos en España, exclu- } \\
\text { yéndolos del sistema de precios máximos. }\end{array}$ \\
\hline 1998 & $\begin{array}{l}\text { Ley } 34 / 1998 \text {, de } 7 \text { de } \\
\text { octubre }\end{array}$ & $\begin{array}{l}\text { Creación de la CNE. } \\
\text { Liberalización de todas las actividades suprimiendo las auto- } \\
\text { rizaciones administrativas vigentes. } \\
\text { Liberalización de todos los precios con excepción del GLP de } 12,5 \mathrm{~kg} \text {. } \\
\text { Se establece el libre acceso a la red logística de productos petro- } \\
\text { líferos. }\end{array}$ \\
\hline
\end{tabular}


CUADRO 2 (continuación)

PRINCIPALES NORMATIVAS LIBERALIZADORAS SOBRE EL SECTOR

(1985-2013)

\begin{tabular}{|c|c|c|}
\hline Año & Normativa & Principales medidas favorecedoras de la competencia \\
\hline 1999 & $\begin{array}{l}\text { Real Decreto-Ley } \\
\text { 15/1999, de } 1 \text { de } \\
\text { octubre }\end{array}$ & $\begin{array}{l}\text { Se obliga a los concesionarios de peajes a la colocación de carteles } \\
\text { informativos en las carreteras estatales sobre tipos, precios y marcas } \\
\text { de carburantes y combustibles petrolíferos ofrecidos en esta- } \\
\text { ciones de servicio, así como también de la distancia a las más } \\
\text { próximas. } \\
\text { Se establece como prioritaria la adjudicación de un área de servicio } \\
\text { en carretera a aquellas propuestas que no impliquen al mismo } \\
\text { suministrador en exclusiva de las estaciones inmediatamente } \\
\text { anterior y posterior. } \\
\text { Establecimiento de la oportunidad de otorgar una nueva concesión } \\
\text { en una misma área de servicio a un competidor del distribuidor ya } \\
\text { instalado. }\end{array}$ \\
\hline 2000 & $\begin{array}{l}\text { Real Decreto-Ley } \\
6 / 2000 \text {, de } 23 \text { de junio }\end{array}$ & $\begin{array}{l}\text { Límite a la integración vertical: Prohibición de incorporar nuevas } \\
\text { estaciones de servicio a la red a operadores con una cuota superior } \\
\text { al } 30 \text { por } 100 \text { durante } 5 \text { años, y, durante tres años a aquellos } \\
\text { operadores que detenten una cuota comprendida entre el } 15 \text { por } \\
100 \text { y el } 30 \text { por } 100 \text { del mercado. } \\
\text { Se obliga a las estaciones de servicio a remitir los precios de venta } \\
\text { al público. } \\
\text { Se establece que los grandes establecimientos comerciales han } \\
\text { de incorporar al menos una instalación de venta de productos } \\
\text { petrolíferos. }\end{array}$ \\
\hline 2001 & $\begin{array}{l}\text { Real Decreto } 114 / 2001 \text {, } \\
\text { de } 9 \text { de febrero }\end{array}$ & Se eliminan las distancias entre estaciones de servicio en carretera. \\
\hline 2001 & $\begin{array}{l}\text { Real Decreto 248/2001, } \\
\text { de } 9 \text { de marzo }\end{array}$ & $\begin{array}{l}\text { Desarrollo del artículo } 7 \text { de la ley 15-1999 sobre carteles en las } \\
\text { carreteras estatales. }\end{array}$ \\
\hline 2013 & $\begin{array}{l}\text { Real Decreto-Ley } \\
4 / 2013 \text {, de } 22 \text { de } \\
\text { febrero }\end{array}$ & $\begin{array}{l}\text { Permite la incorporación de al menos } 1 \text { estación de servicio en zonas } \\
\text { destinadas a actividades comerciales individuales o agrupadas, cent- } \\
\text { ros comerciales, parques comerciales, establecimientos de inspección } \\
\text { técnica de vehículos y zonas o polígonos industriales. } \\
\text { Limitación del contrato vertical al máximo de un año, hasta un } \\
\text { máximo prorrogable de } 3 \text { años. } \\
\text { Prohibición de cláusulas exclusivas que fijen, recomienden o } \\
\text { incidan, directa o indirectamente, en el precio de venta al público } \\
\text { del combustible en los contratos de suministro. }\end{array}$ \\
\hline
\end{tabular}

FUENTE: Elaboración propia en base a CNE (2006) y página web del Ministerio de Industria, Energía y Turismo. 
máximos, no hubo cambio alguno en las 3 legislaturas posteriores, hasta 2013. En este año, el Gobierno aprobó una serie de medidas, auspiciadas en parte por la creciente preocupación pública por el aumento de los precios y acciones anticompetitivas como el denominado «efecto lunes» (véase Jiménez y Perdiguero, 2014).

Entre tales medidas destaca la de permitir la entrada de nuevas estaciones de servicio en polígonos industriales, como una forma de permitir la entrada de nuevos operadores independientes al mercado que, constatadamente, son los que disciplinan la competencia en este sector (Jiménez y Perdiguero, 2012). Este hecho será el que utilizaremos en la sección posterior para tratar de evaluar su impacto en los precios minoristas finales, utilizando para ellos datos detallados del Región Metropolitana de Barcelona.

\section{Evaluación de los efectos del RD 04/2013}

\subsection{Base de datos}

Para aproximar el efecto en los precios de la entrada de nuevas estaciones de servicio a partir del RD 04/2013 disponemos de una base de datos formada por los precios diarios fijados por todas las estaciones de servicio existentes en la Región Metropolitana de Barcelona, desde el dos de enero de 2012 hasta el treinta de junio de 2014 (2 años y medio). El área cuenta con una población, de acuerdo al padrón continuo publicado por la Diputación, de alrededor de 5,03 millones de habitantes.

Los datos de precios van de lunes a viernes y se refieren al precio del combustible diesel después de impuestos. El diesel es el combustible más consumido en España, por lo que resulta el más adecuado para medir el impacto de los nuevos operadores sobre los precios pagados por los consumidores. El precio medio en nuestra base de datos es de 1,395 euros, con un mínimo de 0,95 y un máximo de 1,559, a lo largo de todo el periodo analizado.

Adicionalmente hemos obtenido información sobre la fecha de entrada de cada uno de los nuevos operadores, aproximándola a través del primer día que el nuevo operador remite la información de precios al Ministerio de Industria, Energía y Turismo, tal y como está obligado por Ley. De esta manera podemos conocer la fecha de entrada y por tanto, a partir de qué día los operadores ya establecidos tienen un nuevo competidor.

Además de esto disponemos de la localización geográfica de las estaciones de servicio a través de su longitud y latitud. Esta información nos ha permitido calcular la distancia en metros existente entre cada una de las 579 gasolineras al resto. Por último, disponemos de la enseña comercial de cada una de las gasolineras, lo que nos permitirá distinguir entre la entrada de una gasolinera «low cost», una gasolinera afiliada a una gran superficie o supermercado o si se trata de una gasolinera de una cadena tradicional. Este último argumento es de importancia en el sector, tal y como trabajos anteriores justifican a los operadores independientes como los únicos que disciplinan la competencia en este mercado en España (Jiménez y Perdiguero, 2012). 


\subsection{Análisis econométrico y resultados}

Para tratar de medir el efecto en precios de la entrada de nuevos competidores, diferenciando según la tipología de cada uno de ellos, seguimos la aproximación de Choné y Linnemer (2012), a partir de la cual hemos estimado la siguiente función de precios:

$$
\begin{aligned}
& P_{i t}=\beta_{0}+\beta_{1} \text { Entrante }+\beta_{2} E^{0}+\beta_{3} E^{1}+\beta_{4} E^{2}+\beta_{5} E^{3} \\
& +\beta_{6} E^{4}+\beta_{7} E^{5}+\beta_{8} E^{6}+\beta_{9} E^{7}+\beta_{10} E^{8}+\beta_{11} E^{9}+\varepsilon_{i t}
\end{aligned}
$$

Donde el precio fijado por la gasolinera «i» en el día «t» depende de si es un nuevo entrante o no (variable «Entrante»); de si está situada a menos de un determinado radio $(1.000,1.500$ o 2.000 metros) de alguna de las gasolineras entrantes (variable $\mathrm{E}^{0}$ ) y que consideraremos afectadas de orden 0 ; de si se encuentra a menos de un determinado radio de las afectadas de orden 0 (variable $\mathrm{E}^{1}$ ) y que consideraremos afectadas de orden 1; y así sucesivamente. En el Gráfico 1 se muestra de forma visual la construcción de las diferentes variables que miden la afección de las estaciones de servicio. Además se puede observar que cuando se produce la entrada de una nueva estación de servicio («Entrante»), todas las gasolineras que se encuentran en un radio determinado (1.000, 1.500 o 2.000 metros) las consideraremos afectadas de orden 0 , en nuestro ejemplo gráfico la gasolinera $\langle j »$ y la gasolinera $\langle i »$. Para estas dos gasolineras

\section{GRÁFICO 1}

CONSTRUCCIÓN DE LAS VARIABLES QUE RECOGEN EL EFECTO DE LA ENTRADA DE GASOLINERAS SIGUIENDO A CHONÉ Y LINNEMER (2012)

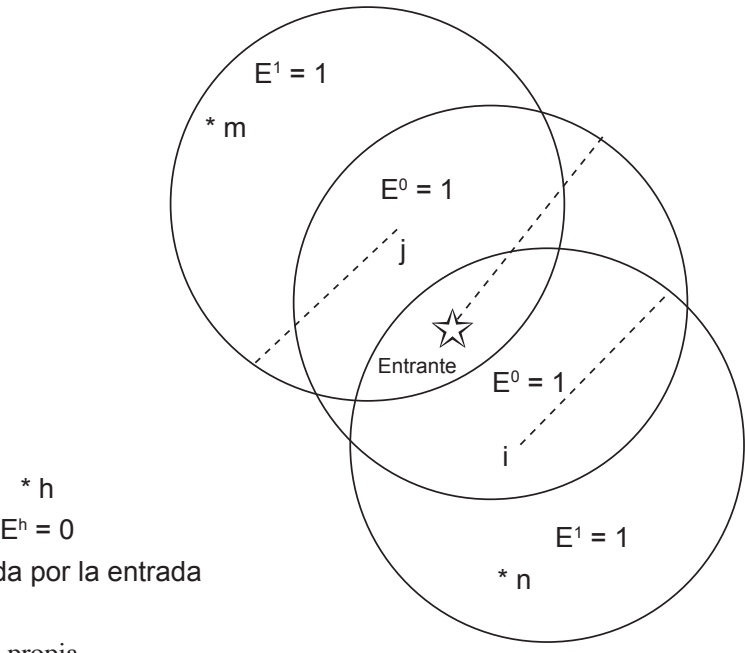

FUENTE: Elaboración propia. 
la variable $\mathrm{E}^{0}$ tomará valor 1 a partir del día en que el «Entrante» comenzó a operar en el mercado. Para el resto de estaciones de servicio el valor de la variable es cero.

Las gasolineras que se encuentran a una mayor distancia del «Entrante», pero que se encuentran dentro del radio de desplazamiento de las gasolineras $« j » e<i\rangle$, las consideraremos afectadas de orden 1 , en nuestro caso las gasolineras «m» $\mathrm{y}\langle n »$. Para estas gasolineras la variable $\mathrm{E}^{1}$ tomará valor 1 desde el día en que el «Entrante» comenzó a operar. El resto de variables $\left(\mathrm{E}^{2}, \mathrm{E}^{3}, \ldots\right)$ se construyen de la misma manera. Aquellas gasolineras que se encuentran a una distancia mayor del radio impuesto a cualquier gasolinera afectada de cualquier grado las consideraremos no afectadas por la entrada, en nuestro ejemplo la gasolinera «h».

En el Cuadro 3 vemos la distribución de gasolineras afectadas por la entrada de algún competidor, distinguiendo si la entrada ha sido de una low cost, de una gasolinera ligada a una cadena de supermercados o a una compañía petrolera tradicional.

Como podemos ver en el Cuadro 3, cuando definimos el radio en 1.000 metros se observa cómo 63 gasolineras están a menos de 1.000 metros de alguna de las

\section{CUADRO 3}

DISTRIBUCIÓN DE ESTACIONES DE SERVICIOS AFECTADAS POR LA ENTRADA DE ALGÚN COMPETIDOR

\begin{tabular}{|c|l|l|l|}
\hline & \multicolumn{1}{|c|}{$\mathbf{1 . 0 0 0}$ metros } & \multicolumn{1}{c|}{$\mathbf{1 . 5 0 0}$ metros } & \multicolumn{1}{c|}{$\mathbf{2 . 0 0 0}$ metros } \\
\hline $\mathrm{E}^{0}$ & $\begin{array}{l}(63 ; 516) \text { Low cost } \\
(9 ; 570) \text { Supermercado } \\
(24 ; 555) \text { Tradicional }\end{array}$ & $\begin{array}{l}(109 ; 470) \text { Low cost } \\
(14 ; 565) \text { Supermercado } \\
(43 ; 536) \text { Tradicional }\end{array}$ & $\begin{array}{l}(146 ; 433) \text { Low cost } \\
(23 ; 556) \text { Supermercado } \\
(62 ; 517) \text { Tradicional }\end{array}$ \\
\hline $\mathrm{E}^{1}$ & $\begin{array}{l}(27 ; 552) \text { Low cost } \\
(4 ; 575) \text { Supermercado } \\
(12 ; 567) \text { Tradicional }\end{array}$ & $\begin{array}{l}(68 ; 511) \text { Low cost } \\
(9 ; 570) \text { Supermercado } \\
(20 ; 559) \text { Tradicional }\end{array}$ & $\begin{array}{l}(88 ; 491) \text { Low cost } \\
(12 ; 567) \text { Supermercado } \\
(42 ; 537) \text { Tradicional }\end{array}$ \\
\hline $\mathrm{E}^{2}$ & $\begin{array}{l}(19 ; 560) \text { Low cost } \\
(2 ; 577) \text { Supermercado } \\
(7 ; 572) \text { Tradicional }\end{array}$ & $\begin{array}{l}(32 ; 547) \text { Low cost } \\
(6 ; 573) \text { Supermercado } \\
(15 ; 564) \text { Tradicional }\end{array}$ & $\begin{array}{l}(38 ; 541) \text { Low cost } \\
(6 ; 573) \text { Supermercado } \\
(30 ; 549) \text { Tradicional }\end{array}$ \\
\hline $\mathrm{E}^{3}$ & $\begin{array}{l}(22 ; 557) \text { Low cost } \\
(1 ; 578) \text { Supermercado } \\
(8 ; 571) \text { Tradicional }\end{array}$ & $\begin{array}{l}(26 ; 553) \text { Low cost } \\
(7 ; 572) \text { Supermercado } \\
(11 ; 568) \text { Tradicional }\end{array}$ & $\begin{array}{l}(14 ; 565) \text { Low cost } \\
(20 ; 559) \text { Tradicional }\end{array}$ \\
\hline $\mathrm{E}^{4}$ & $(6 ; 573)$ Low cost & $\begin{array}{l}(21 ; 558) \text { Low cost } \\
(2 ; 577) \text { Supermercado } \\
(13 ; 566) \text { Tradicional }\end{array}$ & $\begin{array}{l}(4 ; 575) \text { Low cost } \\
(4 ; 575) \text { Tradicional }\end{array}$ \\
\hline $\mathrm{E}^{5}$ & & $\begin{array}{l}(5 ; 574) \text { Low cost } \\
(13 ; 566) \text { Tradicional }\end{array}$ & $\begin{array}{l}(1 ; 578) \text { Low cost } \\
(3 ; 576) \text { Tradicional }\end{array}$ \\
\hline $\mathrm{E}^{6}$ & & $(1 ; 578)$ Low cost & \\
\hline $\mathrm{E}^{7}$ & & $(8 ; 571)$ Tradicional & $(1 ; 578)$ Tradicional \\
\hline $\mathrm{E}^{8}$ & & $(2 ; 577)$ Tradicional & \\
\hline $\mathrm{E}^{9}$ & & $(3 ; 576)$ Tradicional & \\
\hline
\end{tabular}

FUENTE: Elaboración propia a partir de la información del Ministerio de Industria, Energía y Turismo. 
gasolineras low cost que han entrado en el mercado, que serán las afectadas de orden cero y, por tanto, tendrán un valor 1 en la variable $\mathrm{E}^{0}$ a partir del día que comenzó a operar la low cost entrante. Asimismo, podemos observar que con valor 0 para la variable quedan las 516 estaciones que se encuentran a una distancia mayor de la indicada.

Igualmente podemos ver que existen 27 gasolineras que se encuentran a más de mil metros de alguna de las entrantes low cost, pero a menos de esa distancia de alguna de las 63 gasolineras afectadas de orden 0 por una low cost. Estas 27 gasolineras tendrán un valor 1 en la variable $\mathrm{E}^{1}$ a partir del día en que la low cost entrante comenzó a operar. Las 552 restantes tendrán entonces un valor de 0 en $\mathrm{E}^{1}$. Y así sucesivamente para las restantes filas y columnas.

A partir de estos valores descriptivos, en el Cuadro 4 se pueden observar los resultados econométricos obtenidos para la ecuación [1], utilizando un estimador por mínimos cuadrados ordinarios (MCO) con efectos aleatorios.

Como se puede observar en los resultados del Cuadro 4, los nuevos entrantes fijan precios significativamente más reducidos. De hecho, los nuevos competidores low cost fijan precios 10 céntimos más baratos que la media, las gasolineras ligadas a cadenas de supermercados 14 céntimos más baratos que la media, mientras que las gasolineras tradicionales unos 5,5 céntimos.

En el caso de la entrada de una low cost, las que se encuentran entre 1.000 y 2.000 metros, bajan sus precios entre 5,5 y 4,5 céntimos (afectadas de orden cero); las que se encuentran entre 1.000 y 2.000 metros de las afectadas de orden 1, bajan los precios entre 4,2 y 3,7 céntimos (afectadas de orden uno); y el efecto se va diluyendo a medida que nos alejamos geográficamente del punto de entrada.

Por tanto, podríamos concluir que la entrada de nuevos operadores, especialmente de gasolineras low cost y ligadas a cadenas de supermercados, generan descensos significativos en las gasolineras cercanas, lo que provoca un beneficio considerable para los consumidores.

El efecto de la entrada se encadena de una gasolinera a otra, provocando que el efecto se traslade a gasolineras situadas incluso a 10 kilómetros de distancia del nuevo entrante, a pesar de que a medida que la gasolinera está más alejada del punto de entrada, el efecto es cada vez más reducido.

Para controlar cualquier tipo de efecto que pueda afectar a una estación de servicio en particular (diferente de la entrada de nuevos competidores) o algún efecto temporal a todas las estaciones de servicio, se ha realizado la misma estimación utilizando efectos fijos por estación de servicio y por día. Para esta opción estratégica no podemos introducir la variable «Entrada», ya que la combinación lineal de los efectos fijos es igual a esta variable. En el Cuadro 5 se pueden ver los resultados econométricos obtenidos.

Los resultados econométricos utilizando efectos fijos incluidos en el Cuadro 5 no cambian significativamente respecto a los resultados del Cuadro 4, donde utilizábamos efectos aleatorios. Si bien los resultados del test de Hausman no nos permiten aseverar que los estimadores obtenidos usando efectos aleatorios sean 


\section{CUADRO 4 \\ RESULTADOS ECONOMÉTRICOS. ECUACIÓN [1] (MCO y efectos aleatorios)}

\begin{tabular}{|c|c|c|c|}
\hline & 1.000 metros & 1.500 metros & 2.000 metros \\
\hline Entrada Low Cost (L.C.) & $\begin{array}{c}-0,103 * * * \\
(0,005)\end{array}$ & $\begin{array}{c}-0,107 * * * \\
(0,005)\end{array}$ & $\begin{array}{c}-0,108 * * * \\
(0,005)\end{array}$ \\
\hline Entrada Supermercado (S.) & $\begin{array}{c}-0,138 * * * \\
(0,012)\end{array}$ & $\begin{array}{c}-0,143 * * * \\
(0,012)\end{array}$ & $\begin{array}{c}-0,144 * * * \\
(0,012)\end{array}$ \\
\hline Entrada Gasolinera Tradicional (T.) & $\begin{array}{c}-0,050^{* * *} \\
(0,008)\end{array}$ & $\begin{array}{c}-0,054 * * * \\
(0,008)\end{array}$ & $\begin{array}{c}-0,055^{* * *} \\
(0,008)\end{array}$ \\
\hline $\mathrm{E}^{0}$ L.C. & $\begin{array}{c}-0,055^{* * *} * \\
(0,001)\end{array}$ & $\begin{array}{c}-0,048 * * * \\
(0,000)\end{array}$ & $\begin{array}{c}-0,045^{* * *} * \\
(0,000)\end{array}$ \\
\hline $\mathrm{E}^{0} \mathrm{~S}$ & $\begin{array}{c}-0,043 * * * \\
(0,002) \\
\end{array}$ & $\begin{array}{c}-0,049 * * * \\
(0,001)\end{array}$ & $\begin{array}{c}-0,042 * * * \\
(0,001) \\
\end{array}$ \\
\hline $\mathrm{E}^{0} \mathrm{~T}$. & $\begin{array}{c}-0,028 * * * \\
(0,001)\end{array}$ & $\begin{array}{c}-0,029 * * * \\
(0,001)\end{array}$ & $\begin{array}{c}-0,027 * * * \\
(0,001) \\
\end{array}$ \\
\hline $\mathrm{E}^{1}$ L.C. & $\begin{array}{c}-0,042 * * * \\
(0,001)\end{array}$ & $\begin{array}{c}-0,043 * * * \\
(0,001)\end{array}$ & $\begin{array}{c}-0,037 * * * \\
(0,001)\end{array}$ \\
\hline $\mathrm{E}^{1} \mathrm{~S}$. & $\begin{array}{c}-0,050^{* * *} \\
(0,002)\end{array}$ & $\begin{array}{c}-0,036 * * * \\
(0,002)\end{array}$ & $\begin{array}{c}-0,038^{* * *} * \\
(0,001)\end{array}$ \\
\hline $\mathrm{E}^{1} \mathrm{~T}$. & $\begin{array}{c}-0,027 * * * \\
(0,001)\end{array}$ & $\begin{array}{c}-0,017 * * * \\
(0,001)\end{array}$ & $\begin{array}{c}-0,030^{* * *} \\
(0,001)\end{array}$ \\
\hline $\mathrm{E}^{2}$ L.C. & $\begin{array}{c}-0,045^{* * *} \\
(0,001)\end{array}$ & $\begin{array}{c}-0,039 * * * \\
(0,001)\end{array}$ & $\begin{array}{c}-0,034 * * * \\
(0,001)\end{array}$ \\
\hline $\mathrm{E}^{2} \mathrm{~S}$ & $\begin{array}{c}-0,032 * * * \\
(0,003)\end{array}$ & $\begin{array}{c}-0,041 * * * \\
(0,002)\end{array}$ & $\begin{array}{c}-0,027 * * * * \\
(0,002) \\
\end{array}$ \\
\hline $\mathrm{E}^{2} \mathrm{~T}$. & $\begin{array}{c}-0,018^{* * *} \\
(0,001)\end{array}$ & $\begin{array}{c}-0,029 * * * \\
(0,001)\end{array}$ & $\begin{array}{c}-0,024 * * * \\
(0,001)\end{array}$ \\
\hline $\mathrm{E}^{3}$ L.C. & $\begin{array}{c}-0,038^{* * *} \\
(0,001)\end{array}$ & $\begin{array}{c}-0,030 * * * \\
(0,001)\end{array}$ & $\begin{array}{c}-0,028 * * * \\
(0,001)\end{array}$ \\
\hline $\mathrm{E}^{3} \mathrm{~S}$ & $\begin{array}{l}-0,007 \\
(0,004)\end{array}$ & $-0,026 * * *$ & $(0,002)$ \\
\hline $\mathrm{E}^{3} \mathrm{~T}$. & $\begin{array}{c}-0,014 * * * \\
(0,001)\end{array}$ & $\begin{array}{c}-0,034 * * * \\
(0,001)\end{array}$ & $\begin{array}{c}-0,021 * * * \\
(0,001)\end{array}$ \\
\hline $\mathrm{E}^{4}$ L.C. & $\begin{array}{c}-0,058^{* * *} \\
(0,002)\end{array}$ & $\begin{array}{c}-0,034 * * * \\
(0,001)\end{array}$ & $\begin{array}{c}-0,040^{* * *} \\
(0,002)\end{array}$ \\
\hline $\mathrm{E}^{4} \mathrm{~S}$. & & $\begin{array}{c}-0,034 * * * \\
(0,003)\end{array}$ & \\
\hline $\mathrm{E}^{4} \mathrm{~T}$. & & $\begin{array}{c}-0,021 * * * \\
(0,001)\end{array}$ & $\begin{array}{c}-0,012 * * * \\
(0,002)\end{array}$ \\
\hline
\end{tabular}




\section{CUADRO 4 (continuación) \\ RESULTADOS ECONOMÉTRICOS. ECUACIÓN [1] (MCO y efectos aleatorios)}

\begin{tabular}{|c|c|c|c|}
\hline & 1.000 metros & 1.500 metros & 2.000 metros \\
\hline $\mathrm{E}^{5}$ L.C. & $\begin{array}{c}-0,033 * * * \\
(0,004)\end{array}$ & $\begin{array}{c}-0,029 * * * \\
(0,002)\end{array}$ & $\begin{array}{c}-0,047 * * * \\
(0,004)\end{array}$ \\
\hline $\mathrm{E}^{5} \mathrm{~T}$. & & $\begin{array}{c}-0,022^{* * *} * \\
(0,000)\end{array}$ & $\begin{array}{c}-0,033^{* * *} * \\
(0,003)\end{array}$ \\
\hline $\mathrm{E}^{6}$ L.C. & & $\begin{array}{c}-0,025^{* * *} \\
(0,006)\end{array}$ & \\
\hline $\mathrm{E}^{6} \mathrm{~T}$. & & $\begin{array}{c}-0,012^{* * *} \\
(0,001)\end{array}$ & \\
\hline $\mathrm{E}^{7} \mathrm{~T}$. & & $\begin{array}{c}-0,007 * \\
(0,004) \\
\end{array}$ & \\
\hline $\mathrm{E}^{8} \mathrm{~T}$. & & $\begin{array}{c}-0,006^{*} \\
(0,004) \\
\end{array}$ & \\
\hline Constante & $\begin{array}{c}1,365 * * * \\
(0,001)\end{array}$ & $\begin{array}{c}1,369 * * * \\
(0,001)\end{array}$ & $\begin{array}{c}1,370 * * * \\
(0,001)\end{array}$ \\
\hline Observaciones & 256.727 & 256.727 & 256.727 \\
\hline $\mathrm{R}^{2}$ & 0,071 & 0,126 & 0,135 \\
\hline Wald Chi ${ }^{2}$ & $20264,87 * * *$ & $37575,36^{* * *}$ & $40515,42 * * *$ \\
\hline Hausman & $61.49 * * *$ & $139.30 * * *$ & $103.97 * * *$ \\
\hline
\end{tabular}

NOTAS: $* * *$ (significativo al 1 por 100), $* *$ (significativo al 5 por 100) y * (significativo al 10 por 100).

consistentes, el resultado puede deberse también a la baja variación within dentro de la muestra y no necesariamente a que exista correlación entre los efectos aleatorios y el resto de variables explicativas (véase Wooldrige, 2002). En este sentido, realizar la estimación incluyendo efectos fijos, aún cuando los resultados obtenidos sean menos ricos, nos permite confirmar el efecto estimado sobre los precios de los competidores de la entrada de estaciones de servicio low cost o supermercados.

A modo de generalización, podría parecer que este comportamiento generaría una reducción muy significativa sobre el precio medio, pero se debe señalar que el número de entrantes es relativamente reducido. Únicamente entran 27 nuevas gasolineras low cost, 4 estaciones de servicio ligadas a supermercados y 10 de compañías petroleras tradicionales. De hecho si definimos el radio en 1.000 metros, se verán afectadas 247 gasolineras de forma directa o indirecta por la entrada de alguna gasolinera, sobre las 579 existentes (el 45 por 100).

No debemos olvidar que estamos analizando los precios en la Región Metropolitana de Barcelona, zona densamente poblada y además con una densidad de estaciones de servicio más elevada que en la media de España. En zonas con menor densidad de gasolineras estos efectos probablemente serán inferiores. Por tanto, aunque 


\section{CUADRO 5}

RESULTADOS ECONOMÉTRICOS. ECUACIÓN [1]

(MCO y efectos fijos)

\begin{tabular}{|c|c|c|c|}
\hline & 1.000 metros & 1.500 metros & 2.000 metros \\
\hline $\mathrm{E}^{0}$ L.C. & $\begin{array}{c}-0,055^{* * *} \\
(0,001)\end{array}$ & $\begin{array}{c}-0,048^{* * *} \\
(0,000)\end{array}$ & $\begin{array}{c}-0,045^{* * *} \\
(0,000)\end{array}$ \\
\hline $\mathrm{E}^{0} \mathrm{~S}$. & $\begin{array}{c}-0,043^{* * *} \\
(0,002) \\
\end{array}$ & $\begin{array}{c}-0,049 * * * \\
(0,001) \\
\end{array}$ & $\begin{array}{c}-0,042^{* * *} \\
(0,001) \\
\end{array}$ \\
\hline $\mathrm{E}^{0} \mathrm{~T}$. & $\begin{array}{c}-0,029 * * * \\
(0,001) \\
\end{array}$ & $\begin{array}{c}-0,029 * * * \\
(0,001) \\
\end{array}$ & $\begin{array}{c}-0,027^{* * *} \\
(0,001) \\
\end{array}$ \\
\hline $\mathrm{E}^{1}$ L.C. & $\begin{array}{c}-0,042^{* * *} \\
(0,001) \\
\end{array}$ & $\begin{array}{c}-0,043^{* * *} * \\
(0,001) \\
\end{array}$ & $\begin{array}{c}-0,037 * * * \\
(0,001) \\
\end{array}$ \\
\hline $\mathrm{E}^{1} \mathrm{~S}$. & $\begin{array}{c}-0,050^{* * *} \\
(0,002)\end{array}$ & $\begin{array}{c}-0,036^{* * *} \\
(0,002)\end{array}$ & $\begin{array}{c}-0,038^{* * *} \\
(0,001)\end{array}$ \\
\hline $\mathrm{E}^{1} \mathrm{~T}$. & $\begin{array}{c}-0,027 * * * * \\
(0,001)\end{array}$ & $\begin{array}{c}-0,017 * * * \\
(0,001)\end{array}$ & $\begin{array}{c}-0,031 * * * \\
(0,001)\end{array}$ \\
\hline $\mathrm{E}^{2}$ L.C. & $\begin{array}{c}-0,045 * * * \\
(0,001)\end{array}$ & $\begin{array}{c}-0,039 * * * \\
(0,001) \\
\end{array}$ & $\begin{array}{c}-0,034 * * * \\
(0,001) \\
\end{array}$ \\
\hline $\mathrm{E}^{2}-\mathrm{S}$ & $\begin{array}{c}-0,032 * * * \\
(0,003) \\
\end{array}$ & $\begin{array}{c}-0,041 * * * \\
(0,002) \\
\end{array}$ & $\begin{array}{c}-0,027 * * * \\
(0,002)\end{array}$ \\
\hline $\mathrm{E}^{2} \mathrm{~T}$ & $\begin{array}{c}-0,018 * * * \\
(0,001)\end{array}$ & $\begin{array}{c}-0,029 * * * \\
(0,001) \\
\end{array}$ & $\begin{array}{c}-0,024 * * * \\
(0,001)\end{array}$ \\
\hline $\mathrm{E}^{3}$ L.C. & $\begin{array}{c}-0,038 * * * \\
(0,001) \\
\end{array}$ & $\begin{array}{c}-0,030 * * * \\
(0,001) \\
\end{array}$ & $\begin{array}{c}-0,028 * * * \\
(0,001)\end{array}$ \\
\hline $\mathrm{E}^{3} \mathrm{~S}$ & $\begin{array}{l}-0,007 \\
(0,004)\end{array}$ & $\begin{array}{c}-0,026 * * * \\
(0,001) \\
\end{array}$ & \\
\hline $\mathrm{E}^{3} \mathrm{~T}$ & $\begin{array}{c}-0,015 * * * \\
(0,001)\end{array}$ & $\begin{array}{c}-0,035^{* * *} \\
(0,001)\end{array}$ & $\begin{array}{c}-0,022 * * * \\
(0,001)\end{array}$ \\
\hline $\mathrm{E}^{4}$ L.C. & $\begin{array}{c}-0,057 * * * \\
(0,002)\end{array}$ & $\begin{array}{c}-0,035^{* * *} * \\
(0,001)\end{array}$ & $\begin{array}{c}-0,040 * * * \\
(0,002)\end{array}$ \\
\hline $\mathrm{E}^{4} \mathrm{~S}$ & & $\begin{array}{c}-0,034 * * * \\
(0,003)\end{array}$ & \\
\hline $\mathrm{E}^{4} \mathrm{~T}$ & & $\begin{array}{c}-0,021 * * * \\
(0,001)\end{array}$ & $\begin{array}{c}-0,012 * * * \\
(0,002)\end{array}$ \\
\hline E5 L.C. & $\begin{array}{c}-0,033 * * * \\
(0,004)\end{array}$ & $\begin{array}{c}-0,029 * * * \\
(0,002)\end{array}$ & $\begin{array}{c}-0,046^{* * *} \\
(0,004)\end{array}$ \\
\hline
\end{tabular}




\section{CUADRO 5 (continuación) \\ RESULTADOS ECONOMÉTRICOS. ECUACIÓN [1] \\ (MCO y efectos fijos)}

\begin{tabular}{|c|c|c|c|}
\hline & 1.000 metros & 1.500 metros & 2.000 metros \\
\hline $\mathrm{E}^{5} \mathrm{~T}$ & & $\begin{array}{c}-0,022^{* * *} * \\
(0,001)\end{array}$ & $\begin{array}{c}-0,033^{* * *} * \\
(0,003)\end{array}$ \\
\hline $\mathrm{E}^{6}$ L.C. & & $\begin{array}{c}-0,025^{* * *} * \\
(0,006)\end{array}$ & \\
\hline $\mathrm{E}^{6} \mathrm{~T}$. & & $\begin{array}{c}-0,012 * * * \\
(0,001)\end{array}$ & \\
\hline $\mathrm{E}^{7} \mathrm{~T}$. & & $\begin{array}{c}-0,007 * * \\
(0,004)\end{array}$ & \\
\hline E. T. & & $\begin{array}{c}-0,007 * \\
(0,004)\end{array}$ & \\
\hline Constante & $\begin{array}{c}1,363 * * * \\
(0,000)\end{array}$ & $\begin{array}{c}1,368 * * * \\
(0,000)\end{array}$ & $\begin{array}{c}1,369 * * * \\
(0,000)\end{array}$ \\
\hline Observaciones & 256.727 & 256.727 & 256.727 \\
\hline $\mathrm{R}^{2}$ & 0,0714 & 0,1264 & 0,1351 \\
\hline Wald Chi ${ }^{2}$ & $1.407,38^{* * *}$ & $1.684,30 * * *$ & $2.667,49 * * *$ \\
\hline Hausman & $61,49 * * *$ & $139,30 * * *$ & $103,97 * * *$ \\
\hline
\end{tabular}

NOTAS: *** (significativo al 1 por 100), ** (significativo al 5 por 100) $\mathrm{y} *$ (significativo al 10 por 100). L.C. (Low cost), S. (Supermercado), T (Gasolinera Tradicional).

la entrada de nuevos operadores puede generar descensos de precios significativos en zonas muy locales, en zonas con densidades de gasolineras bajas, la entrada de nuevos operadores puede generar efectos más reducidos.

Igualmente hay que destacar que en la presente aproximación hemos considerado la entrada como un elemento exógeno, pero puede no serlo. Si los entrantes se sitúan principalmente en las zonas más caras, nuestras estimaciones podrían estar sobrevalorando los efectos de la entrada.

Sin embargo, hay que tener en cuenta que las gasolineras no se pueden localizar donde quieran, por lo que aunque existan precios elevados en alguna zona es probable que los operadores (principalmente los low cost) no puedan localizarse. De hecho, todas las estaciones de servicio low cost que entran en el mercado lo hacen en polígonos industriales, lugares donde la legislación les permite entrar, pero quizás no donde elegirían entrar si el acceso fuera libre en cualquier tipo de localización. Por tanto, si existiera ese sesgo presuponemos que no sería lo suficientemente importante como para invalidar las principales conclusiones extraídas en el presente trabajo. 


\section{Conclusiones}

Las actividades del sector público en España conducentes a la liberalización del sector de los carburantes parecen no haber alcanzado los resultados de competencia efectiva que cabrían esperar. Este resultado, compartido en buena parte de las economías modernas, hacen que la actividad pública sectorial se centre en dos cuestiones: detectar los casos de prácticas anticompetitivas $\mathrm{y}$, mediante las sanciones, disuadir de todo tipo de réplicas; y por otra parte, establecer mecanismos legales que faciliten la apertura del mercado.

En este trabajo hemos descrito ambas políticas en las dos últimas décadas para, posteriormente, evaluar econométricamente los efectos de la más reciente de las medidas tomadas por el Gobierno, relativa a la entrada de nuevos operadores minoristas.

Utilizando datos diarios de precios (de lunes a viernes) para el periodo enero de 2012 a junio de 2014, de la Región Metropolitana de Barcelona, planteamos una ecuación de precios para tratar de mostrar el comportamiento en esta materia de los entrantes.

Nuestros resultados apuntan a que las estaciones de servicio denominadas low cost y los supermercados tienen precios más bajos que la media de competidores en su entorno y, además, disciplinan no sólo a las empresas inmediatamente circundantes sino hasta un radio de 10 kilómetros (aunque con reducciones cada vez menores conforme aumenta la distancia). Resultados aparentemente positivos que deben ser tomados con cautela, dado el supuesto de exogeneidad de la entrada y de las características de alta densidad de población de la zona analizada, por lo que los resultados podrían ser ligeramente diferentes en otras zonas de España.

\section{Referencias bibliográficas}

[1] BELLO, A. y CAVERO, S. (2008): «The Spanish retail petroleum market: New patterns of competition since the liberalization of the industry». Energy Policy 36, pp. 612-26.

[2] CHONÉ, P., y LINNEMER, L. (2012) «A treatment effect method for merger analysis with an application to parking prices in Paris». The Journal of Industrial Economics 60(4), pp. 631-56.

[3] COMISIÓN NACIONAL DE LA COMPETENCIA (2012): Informe de seguimiento del mercado de distribución de carburantes de automoción en España.

[4] COMISIÓN NACIONAL DE LA ENERGÍA (2013): Informe sobre el efecto del día de la semana en la determinación de los precios de los carburantes (periodo 2007-2012).

[5] ECKERT, A. (2013): «Empirical studies of gasoline retailing: a guide to the literature». Journal of Economic Surveys, 27(1), pp. 140-166.

[6] IVALDI, M. JULLIEN, B. REY, P., SEABRIGHT, P. y TIROLE, J. (2003): «The Economics of Tacit Collusion». IDEI-Toulouse. Final Report for DG Competition. European Commission.

[7] JIMÉNEZ, J. L. y PERDIGUERO, J. (2012): «Does rigidity of prices hide collusion?». Review of Industrial Organization, 41, pp. 223-248. 
[8] JIMÉNEZ, J. L. y PERDIGUERO, J. (2013): «One more lie: the «Monday effect» in the Spanish retail petrol market». Mimeo.

[9] JIMÉNEZ, J. L. y PERDIGUERO, J. (2014): «Información, presión institucional y estrategias empresariales: el efecto lunes en el mercado español de gasolina». Información Comercial Española. 50 Aniversario de la Primera Ley de Competencia en España, 876, pp. 37-56.

[10] PERDIGUERO, J. (2012): «Tres décadas de reformas en el mercado español de gasolinas: Historia de un fracaso anunciado». Papeles de Economía Española, 134, pp. 143-157.

[11] PERDIGUERO, J. y JIMÉNEZ, J.L. (2009): «¿Competencia o colusión en el mercado de gasolina? Una aproximación a través del parámetro de conducta», Revista de Economía Aplicada, 50 (XVII), pp. 27-45.

[12] WOOLRIDGE, J. M. (2002): Econometric analysis of cross section and panel data, Cambridge, Mass., MIT Press. 